\title{
La Revista Latinoamericana de Psicología en sus 40 años de historia: 1969-2009*
}

\section{Latin American Journal of Psychology: Four Decades of History: 1969-2009}

\begin{tabular}{l|l|l} 
Recibido: junio 7 de 2010 ～Revisado: agosto 28 de 2010 Aceptado: septiembre 1 de 2010
\end{tabular}

\author{
Miguel Gallegos** \\ Facultad de Psicología / Universidad Nacional \\ de Rosario \\ Consejo Nacional de Investigaciones Científicas \\ y Técnicas (UNR-CONICET)
}

Para citar este artículo. Gallegos, M. (2010). La

Revista Latinoamericana de Psicología en sus 40 años de historia: 1969-2009. Universitas Psychologica, 9 (3), 911-924.

* El autor agradece a Rubén Ardila por la información proporcionada para este trabajo.

** Correspondencia con el autor: Bv. 27 de Febrero 210 bis. Código Postal: 2000 Rosario, Santa Fe, Argentina.E-mail: maypsi@yahoo.com.ar

RESUMEN

Se presenta un trabajo que revisa la historia de la Revista Latinoamericana de Psicología (RLP) durante las cuatro décadas de existencia: 1969-2009. Desde el inicio, la RLP ha sostenido su calidad editorial, tres números anuales, contenidos con diversas perspectivas teóricas y metodológicas, autores procedentes de diferentes países del mundo, entre otras cosas. En el desarrollo del trabajo, se puede encontrar una breve historia de las motivaciones e intereses del fundador de la revista, una descripción del formato editorial y la presentación de varios indicadores que reflejan la importancia de la revista en el contexto latinoamericano e internacional. Sabiendo que muy pocas revistas de psicología, originadas en el contexto latinoamericano, han logrado superar la barrera del tiempo y la calidad editorial, es un grato honor llegar a celebrar 40 años de historia de la RLP.

Palabras clave autor

Revista Latinoamericana de Psicología, historia, psicología, América Latina.

Palabras clave descriptor

FALTA

\section{A B S T R A C T}

This article reviews the history of Latin American Journal of Psychology (RLP) in the last four decades (1969-2009). From the beginning, the RLP has sustained its editorial quality, three numbers a year, different theoretical perspectives and methodologies, authors from different countries, etc. Original editor's motivations and interests are presented, also editorial indicators descriptions are analyzed showing the relevance of the Journal both in Latin American and international context. It is important to say that a few number of Journals in Psychology in Latin American context could had a long time of outcome and editorial quality as the RLP.

Keywords author

Latin American Journal of Psychology, history, psychology, Latin America.

Keywords plus

FALTA 


\section{Introducción}

En la historia de la psicología en América Latina, se puede encontrar una importante cantidad de revistas de psicología. Algunas revistas, que se gestaron desde principios de siglo XX, se pueden caracterizar como publicaciones afines: Archivos de Psiquiatría y Criminología (1902), Archivos de Pedagogía y Ciencias Afines (1906) y Revista de Filosofía (1915) en Argentina; Revista de Psiquiatría y Disciplinas Conexas (1918) y Revista de Neuro-Psiquiatría (1938) en Perú; Archivos Brasileiros de Hygiene Mental (1923), Annaes da Colônia de Psychopathas (1928) y Educação (1928) en Brasil, entre otras.

Otras publicaciones más cercanas al tiempo de creación de las carreras de psicología, alrededor de 1950, se pueden caracterizar como publicaciones de transición: Anales del Instituto de Psicología (1935) y Anales del Instituto de Investigaciones Psicopedagógicas (1952) en Argentina; Archivos del Instituto de Psicología (1941) en Chile; Boletín del Instituto Psicopedagógico Nacional (1942) en Perú; Hoja de Psicología (1947) en Uruguay; Arquivos Brasileiros de Psicotecnia (1949)² en Brasil, entre otras.

También pueden señalarse las publicaciones propiamente psicológicas, en las que han participado los primeros titulados psicólogos en las respectivas laborales editoriales: Revista Mexicana de Psicología (1963)³; Revista Venezolana de Psicología (1962); Jornal Brasileiro de Psicologia (1964); Revista Argentina de Psicología (1969); Revista Boliviana de Psicología (1975); Revista Uruguaya de Psicología (1978); Revista Chilena de Psicología (1978); Revista Costarricense de Psicología (1982); Revista Cubana

1 Esta publicación también fue difundida como Trabalhos de Psychologia, aunque solamente se editaron dos números en 1928 y 1929 (véanse Centofanti, 1982; León, 1997).

2 A partir de 1969 fue denominada Arquivos Brasileiros de Psicologia Aplicada (1969) y a partir de 1979 Arquivos Brasileiros de Psicologia (Guedes \& Campos, 1999; Lemos, 2005).

3 En 1984, la Sociedad Mexicana de Psicología volvió a fundar otra revista con el mismo título, aunque sin ninguna continuidad con la anterior (Aguilera, 2006; Ardila, 2010). de Psicología (1984) ${ }^{4}$; Revista Colombiana de Psicología $(1992)^{5}$, entre otras.

Asimismo, es posible identificar varias revistas que surgieron motivadas por lograr un impacto más allá de las fronteras nacionales de cada país. Estas revistas serían las primeras publicaciones de alcance regional o internacional: Acta Psiquiátrica y Psicológica de América Latina $(1964)^{6}$; Revista Interamericana de Psicología (1967); Aprendizaje y Comportamiento (1978); Avances en Psicología Clínica Latinoamericana $(1982)^{7}$; Revista Interamericana de Psicología Ocupacional (1982); Archivo Latinoamericano de Historia de la Psicología y Ciencias Afines (1988); Revista Intercontinental de Psicología y Educación (1988), entre otras.

Lógicamente, se trata de un conjunto de publicaciones bastante heterogéneo y polimorfo, tanto en las orientaciones temáticas como en los propósitos editoriales. Salvo algunas excepciones, la mayoría de estas revistas ha tenido una serie de dificultades comunes: baja calidad editorial, escasa circulación nacional e internacional, nacimientos pocos planificados, desapariciones prematuras, discontinuidades en los números, entre otras (Ardila, 1969a, 1986a, 1988, 1999).

Dentro del amplio espectro de las publicaciones psicológicas, merece destacarse la Revista Latinoamericana de Psicología (RLP), que ha llegado a cumplir más de 14.600 días de existencia. Durante cuatro décadas se ha editado ininterrumpidamente, contribuyendo con la difusión de la psicología como ciencia y profesión. Fundada en agosto de

4 Antes de esta publicación, ya se reconocía la existencia de una primera Revista Cubana de Psicología en 1955, aunque tuvo una corta vida (Dueñas, 2005).

5 Esta publicación se propuso continuar con la labores de la Revista de Psicología, iniciada en 1956 (López, 1992). Sin embargo, en la práctica, fue más bien un reemplazo (Ardila, 2010; González, 1999).

6 Esta publicación se inició con el nombre de Acta Neuropsiquiátrica Argentina en 1954, luego fue reconvertida en Acta Psiquiátrica y Psicológica Argentina en 1962 y, más tarde, recibió su denominación actual (véanse Amil \& Jardón, 2007; Kirsch \& Rodríguez Sturla, 2006; Kirsch \& Rojas Breu, 2007; Klappenbach et al., 1999; Rossi, 2006, 2007, 2008).

7 Esta revista fue gestada por Rubén Ardila en 1982 (Calvache, López López \& Mayorga, 2002); desde el 2004 se denomina Avances en Psicología Latinoamericana (Pérez-Acosta, 2008). 
$1968^{8}$ y editada a partir de enero de 1969 (Ardila, 1998), la RLP no sólo se propuso ser una canal de información científica para la psicología, sino también "un puente de unión entre los psicólogos de nuestro continente" (Ardila, 1969b).

En cierto modo, la historia de la psicología como profesión en América Latina, que se inicia alrededor de la década de 1950, es casi coetánea de la historia de la RLP. Un breve repaso de su historia implicaría recordar a su gestor y sus motivaciones, la estructura editorial y algunos indicadores de su presencia en la comunidad psicológica.

\section{Rubén Ardila y la fundación de la RLP}

Detrás de toda iniciativa de publicación existen diferentes motivaciones e intereses. En el caso particular de la RLP, esta iniciativa no hubiese sido posible sin la intervención y motivación de Rubén Ardila, quien tuvo la ambiciosa idea de gestar una publicación de psicología con alcance internacional, en un tiempo que seguramente muy pocos creían en la prosperidad de esta idea. Sin embargo, a pesar de los pronósticos, la RLP llegó a plasmarse y tener una larga continuidad. Los frecuentes editoriales de su creador así lo fueron testimoniando (véase Ardila, 1973, 1978, 1979, 1986b, 1988, 1998, 2004, 2007).

La idea de crear una publicación como la RLP, fue concebida a propósito de la asistencia de Rubén Ardila al XI Congreso Interamericano de Psicología, que tuvo lugar en México, en 1967 (Ardila, 1986b). En ese tiempo, Rubén Ardila tenía 25 años y estaba realizando sus estudios de posgrado en la Universidad de Nebraska (López López, 2003).

Durante el citado evento, Rubén Ardila había detectado un gran vacío y desconocimiento acerca de lo que hacían los psicólogos en América Latina. Para aquel entonces, se conocían ampliamente los autores, las teorías y las metodologías de trabajo de la psicología en Norteamérica y Europa, pero

8 En esa fecha se realizaron las gestiones administrativas (registro y pedido de ISSN: 0120-0534) y académicas (conformación del comité editorial, contacto con posibles autores, etc.) para poner en funcionamiento la edición de la revista (Ardila, 2010). poco se sabía de la psicología en la región (Ardila, 1978, 1986b, 1998). Fue a partir de este estado de vacío y desconocimiento que Rubén Ardila decidió emprender la iniciativa de la RLP.

A esta historia documentada de los orígenes de la RLP, puede agregarse una anécdota tal vez poco conocida. El primer número de la RLP fue costeado con un dinero que los padres de Rubén Ardila le obsequiaron para su matrimonio. El propio Ardila ha manifestado que la RLP tuvo problemas de financiación en sus inicios, pero logró componerse con el tiempo, hasta llegar a ser totalmente autofinanciada (Ardila, 1986b).

La RLP ha zanjado toda un historia de la psicología en América Latina, que se yergue a partir de la profesionalización de la psicología en la década de 1950. Rubén Ardila, su fundador, un "psicólogo latinoamericano" (Ardila, 1994), fue uno de los primeros psicólogos formados en estas tierras que se propuso inaugurar, entre otros cosas, una publicación científica de alcance regional9. Con toda justicia puede ser caracterizado como uno de los pioneros de la psicología en América Latina.

$\mathrm{Si}$ a otros actores no psicólogos les ha tocado ser bautizados como los pioneros (Ardila, 1986a; García, 2007, 2008; Salas \& Lizama, 2009), sin dudas, Rubén Ardila puede ser nominado como uno de los pioneros de la segunda camada. Cuando se escriba la historia de la segunda generación de pioneros, seguramente el nombre de Rubén Ardila va a tener un lugar destacado ${ }^{10}$.

9 Otros datos biográficos y académicos de Rubén Ardila pueden consultarse en: Ardila (1994); Flórez (2003); Pérez-Acosta (2007).

10 La Sociedad Interamericana de Psicología está preparando un libro con diversas autobiografías de psicólogos iberoamericanos. En este libro, que compilan Hugo Klappenbach y Ramón León, se presentan las autobiografías de Julio Villegas (Chile), Reynaldo Alarcón (Perú), Rogelio Díaz-Guerrero (México), Arrigo Angelini (Brasil), Helio Carpintero (España) y Rubén Ardila (Colombia), entre otros. Es probable que ese libro pueda ser una buena representación de lo que llamamos "la segunda generación de pioneros" (Gallegos, 2009). 


\section{La estructura editorial de la RLP}

Se ha dicho que la RLP surgió para dar lugar a la difusión de la psicología en los países de América Latina. Sin embargo, no se redujo a ser una publicación estrictamente cerrada a la región, sino, además, se propuso mantener una recepción y alcance internacional. Tanto es así que las colaboraciones podían remitirse desde cualquier parte del mundo en español, portugués, inglés, francés, etc.

Los trabajos se publicaban mayoritariamente en español con el resumen en inglés (Ardila, 1969b). Sólo en muy pocas ocasiones se publicaron trabajos en otros idiomas distintos al español. En estos casos, los trabajos se traducían al español con el consentimiento del autor (R., Ardila, comunicación personal, 2010, febrero 14). Recién en el último tiempo, han comenzado a incluirse con mayor frecuencia trabajos en inglés.

Primariamente, la RLP fue una iniciativa particular de su fundador. Cuando se crea la Fundación para el Avance de la Psicología en 1977, la RLP se institucionaliza. A partir de allí, sería una publicación gestionada administrativa y académicamente por esta institución, hasta que en el año 2007 pasa a ser editada por la Fundación Universitaria Konrad Lorenz (Ardila, 2007).

La RLP comenzó organizada editorialmente en cuatro secciones: artículos, comentarios, libros y el mundo de la psicología (Ardila, 1969b). La sección artículos recibía trabajos en todas las áreas de la psicología, que representaran una contribución original a la psicología como ciencia y profesión. La sección comentarios incluía reflexiones y críticas de orden general. La sección libros contenía las reseñas de textos publicados recientemente, y la sección el mundo de la psicología reunía un conjunto de noticias e informaciones ligadas a la psicología.

Con el tiempo, la RLP ha mantenido su organización editorial. Sin embargo, la sección comentarios fue perdiendo vitalidad y, en cambio, la sección referida al mundo de la psicología fue ganando espacio. En esta sección, se han publicado importantes ensayos, reseñas históricas, biografías y otras informaciones. Las noticias biográficas de autores representativos de la psicología han sido una constante (Anónimo, 1998; Ardila, 1988; Gutiérrez, Pérez-Acosta \& Plata-Caviedes, 2009).

Uno de los agregados que comenzó a incluirse periódicamente a partir de 1970. fue la edición de los números monográficos. Estos números temáticos, de gran importancia para el acervo de conocimientos de una disciplina (Sanabria, 1998), son organizados por un editor invitado, quien asume la responsabilidad de solicitar las contribuciones y se encarga de la revisión de los artículos (Ardila, 1978).

En las últimas páginas de la RLP, siempre se incluían noticias de instituciones, anuncios de congresos, auspicios de revistas y eventualmente alguna publicidad. Durante muchos años, esta información sirvió para tomar conocimiento de las actividades científicas y profesionales, que se desarrollaban en distintas parte del mundo.

Sin dudas, aquellas páginas reporteras cumplieron satisfactoriamente con su labor, en un tiempo en el que la circulación de información no era tan dinámica e instantánea como en el presente. En la actualidad, estas informaciones han sido canceladas, dado que existen otros medios más idóneos y eficaces (Gutiérrez, Pérez-Acosta \& Plata-Caviedes, 2009).

Lógicamente, el editor responsable de la RLP fue Rubén Ardila, quien se desempeñó en el cargo durante 35 años (Ardila, 2004). Desde el 2004, continuaron con la tarea Germán Gutiérrez como editor y Andrés Pérez-Acosta como editor asociado; ambos ya venían integrando el cuerpo editorial de la revista desde hace años. A partir del 2008, se hizo cargo de la edición Marithza Sandoval, y desde el 2010 Luisa Fernanda Ramírez, ambas pertenecientes a la Fundación Universitaria Konrad Lorenz.

Demás está decir que, al ser una edición científica, todos los artículos publicados son arbitrados por pares evaluadores y las normas de publicación siguen el manual de estilo de la American Psychological Association. Quienes han oficiado de evaluadores se encuentran entre los profesionales más renombrados y destacados de la psicología en América Latina y de otros países del mundo (Gutiérrez, 2008; Gutiérrez et al., Pérez-Acosta $\&$ Plata-Caviedes, 2009). 
A lo largo de su historia, siempre se han editado tres números por año, salvo en escasas oportunidades donde se combinaron dos números en una edición. El formato de publicación siempre ha sido el impreso. En la actualidad, el formato impreso se combina con el formato electrónico; por tanto, todo el contenido de la RLP puede ser leído online y sin restricciones desde el primer número publicado.

Las fluctuaciones socioeconómicas de Colombia, país editor, y la variación de la cantidad de suscripciones en los distintos períodos han condicionado la cantidad de ejemplares editados. En un comienzo, se editaron alrededor de 1.000 ejemplares por números, luego 1.500 (Ardila, 1986b) y, más tarde, el tiraje se estabilizó en 2.500 ejemplares (Gutiérrez et al., 2009). Una proporción considerable de ejemplares se enviaban, sin cargo, a distintas instituciones y representantes clave de la psicología en el mundo (Ardila, 1986b).

La actual institución editora ha implementado un sistema de cobro por artículo, que oscila entre los 350 y 500 dólares, para cubrir los costos de edición de la revista. Se trata de una nueva política editorial que tiende a buscar una mejor autofinanciación, suplantando la vieja política de estar suscripto a la revista para poder someter artículos a evaluación. Esta política trata de comprometer a las instituciones, en la financiación de los costos de la difusión del conocimiento, sin que recaiga el peso económico en los investigadores (Sandoval, 2009).

\section{Algunos indicadores de la RLP}

La RLP nunca ha tenido problemas por la escasez de artículos, siempre ha recibido más contribuciones de las que realmente puede publicar; esto ha redundado en la calidad del contenido publicado (Ardila, 1978, 1986b, 1988). De acuerdo a ciertos estudios bibliográficos, es posible sintetizar algunos parámetros cuantitativos de la RLP, en sus 40 años de edición:
Resumen de algunos indicadores de la RLP 1969. 2008

\begin{tabular}{lc}
\hline Cantidad de artículos & 922 \\
$\begin{array}{l}\text { Cantidad de monográ- } \\
\text { ficos }\end{array}$ & 33 \\
Cantidad de biografías & 86 \\
Cantidad de reseñas de & 1.200 \\
libros & (aproximadamente) \\
\hline
\end{tabular}

Fuente: elaboración propia

Durante la primera década (1969-1978), se publicaron 234 artículos (Ardila, 1978); en la segunda década (1979-1988), se publicaron 237 artículos (Quiñones et al., 1991; Vera, Quiñones, Sánchez \& Pedraja, 1991); en la tercera década (1989-1998), se publicaron 206 artículos (López López \& Calvache, 1998); y, en la cuarta década (1999-2008), se publicaron 254 artículos (Gutiérrez et al., 2009). Esto hace un total de 922 artículos publicados durante las cuatros décadas (1969-2008), aunque otros autores reporten 925 artículos (Gutiérrez et al., 2009).

Para las primeras dos décadas, se ha informado una tasa de rechazo de los artículos recibidos cercana al 60\%; siempre informando al autor de las razones del mismo (Ardila, 1978b, 1988). Por tanto, se publicaba alrededor del $40 \%$ de los trabajos evaluados. En tiempos más recientes, la tasa de rechazo se ha estimado entre el $70 \%$ y $80 \%$ (Gutiérrez et al., 2009).

En todos estos años de labor editorial continua, se han publicado 33 monográficos, con énfasis en diversas áreas de la psicología. Por año, incluyendo los tres números, se han publicado alrededor de 30 reseñas de libros (Gutiérrez et al., 2009). Entretanto, la cantidad de biografías de psicólogos y psicólogas incluidas en la revista, llegaron a 86.

Aun reconociendo que la RLP es una revista que publica trabajos en todas las áreas de la psicología, no obstante, se pueden visualizar algunas preferencias temáticas. Ardila (1978) ha informado que los trabajos clasificados en el área de la psicología social y en la de la psicología del aprendizaje 
(modificación del comportamiento), tuvieron una presencia importante, durante el primer decenio de rodaje de la revista.

Para la segunda década, el área de la psicología social seguía manteniendo el liderazgo, aunque también se publicaron una cantidad importante de trabajos en las áreas de la psicología evolutiva, la biorretroalimentación, la psicología gerontológica, la psicología clínica, la psicología educativa y la psicología comparada (Quiñones et al., 1991).

En la tercera década, la mayoría de los trabajos se incluyeron en el área de la psicología educativa, seguida muy de cerca por el área de la psicología social y el área de la psicología evolutiva. No menos protagónicas fueron las áreas de la psicología clínica, la psicología de la salud e historia de la psicología y sistemas (López López \& Calvache, 1998).

En su conjunto, durante las cuatro décadas, las grandes áreas temáticas de la psicología social y del análisis del comportamiento, fueron las que más contribuciones aportaron. Sin embargo, ambas áreas reunidas representaron algo más del 25\% total de las contribuciones temáticas publicadas. El resto se distribuye en los diferentes campos de investigación y aplicación de la psicología (Gutiérrez et al., 2009).

Es obvio que la inclusión de los diversos números monográficos, hizo que algunas áreas tuvieran más presencia de artículos que otras, y esto haya reflejado alguna variación en los distintos períodos. Los 33 números monográficos que se han publicado hasta el momento, de acuerdo a ciertas categorizaciones previas, son:
Números monográficos de la RLP

\begin{tabular}{|c|c|}
\hline $\begin{array}{l}\text { Psicología } \\
\text { como cien- } \\
\text { cia }\end{array}$ & $\begin{array}{l}\text { Aprendizaje temprano } \\
\text { Comportamiento sexual humano } \\
\text { Psicología evolutiva } \\
\text { Psicología comparada } \\
\text { Percepción y psicofísica } \\
\text { Personalidad } \\
\text { La síntesis experimental del comporta- } \\
\text { miento } \\
\text { Psicología social } \\
\text { Investigación básica } \\
\text { Análisis experimental del comporta- } \\
\text { miento y problemas sociales } \\
\text { Psicología de la adolescencia } \\
\text { Análisis conductual del lenguaje y la } \\
\text { cognición } \\
\text { Neurociencias del comportamiento }\end{array}$ \\
\hline $\begin{array}{l}\text { Campos de } \\
\text { aplicación } \\
\text { de la psico- } \\
\text { logía }\end{array}$ & $\begin{array}{l}\text { Terapia del comportamiento } \\
\text { Psicología industrial y organizacional } \\
\text { Psicología educacional } \\
\text { Psicología clínica } \\
\text { Psicología gerontológica } \\
\text { Biorretroalimentación } \\
\text { Psicología de la salud } \\
\text { El mundo del trabajo } \\
\text { Psicología política } \\
\text { Problemas sociales } \\
\text { Psicología y sida } \\
\text { Educación y psicología } \\
\text { Ciclo vital, envejecimiento y vejez }\end{array}$ \\
\hline $\begin{array}{l}\text { La psicolo- } \\
\text { gía en Lati- } \\
\text { noamérica }\end{array}$ & $\begin{array}{l}\text { Psicología experimental latinoameri- } \\
\text { cana } \\
\text { Psicología en el Perú } \\
\text { Psicología en Puerto Rico } \\
\text { Psicología en Guatemala }\end{array}$ \\
\hline $\begin{array}{l}\text { Números } \\
\text { conmemo- } \\
\text { rativos }\end{array}$ & $\begin{array}{l}\text { El siglo de Vygotsky y de Piaget } \\
20 \text { años } \\
\text { Los primeros } 30 \text { años de la Revista La- } \\
\text { tinoamericana de Psicología }\end{array}$ \\
\hline
\end{tabular}

Fuente: Sanabria (1998); Gutiérrez, Pérez-Acosta y PlataCaviedes (2009).

Durante los primeros 30 años, el tipo de investigación más reportada en los artículos publicados fue la empírica (49\%), seguida muy de cerca por las producciones teóricas (41\%). Menos protagónicos fueron los trabajos psicométricos $(5 \%)$ y documentales (3\%). Vale destacar que dentro de 
las investigaciones empíricas, los sujetos o participantes más utilizados fueron los humanos en un 90\%, y los animales en un 10\% (López López \& Calvache, 1998).

Básicamente, la RLP ha mantenido un buen balance de publicación entre trabajos teóricos y trabajos empíricos. Sin embargo, la tendencia general durante las 40 décadas, ha sido la de publicar más trabajos empíricos que teóricos. Para algunos autores, esta tendencia representa el interés de la revista por estimular la producción de nuevos conocimientos (Gutiérrez et al., 2009).

Algunos análisis bibliográficos han permitido conocer ciertas características de la RLP. En general, la cienciometría y los estudios bibliométricos están constituyendo verdaderas herramientas, para el análisis y el procesamiento de la información científica, permitiendo evaluar tanto el desempeño de los establecimientos académicos como el rendimiento de las publicaciones científicas, a través de diversos indicadores (Carpintero \& Peiró, 1981; Filippo \& Fernández, 2002; Lascurain, López \& González, 1997; López, 1996; López López, 2009; Macías-Chapula, 1998; Rousseau, 1997).

Con base en estos estudios, se han podido identificar los autores, los libros y las revistas, más citados, en las páginas de la RLP. En cuanto a los autores más citados en los artículos publicados, sin contemplar las autocitas, para las tres primeras décadas, se pueden destacar los siguientes.

Autores más citados en el período 1969-1998

\begin{tabular}{lcc}
\hline \multicolumn{1}{c}{ Autores } & № Citas & \% \\
\hline Skinner, B. F. & 132 & 0.79 \\
Eysenck, H. J. & 88 & 0.52 \\
Piaget, J. & 74 & 0.44 \\
Ardila, R. & 58 & 0.34 \\
Bandura, A. & 58 & 0.34 \\
Díaz-Guerrero, R. & 47 & 0.28 \\
Vygotsky, L. S. & 46 & 0.27 \\
Delgado, H. & 44 & 0.26 \\
Otros Autores & 16.122 & 96.71 \\
\hline
\end{tabular}

Fuente: López López y Calvache (1998).
Para el mismo período, se pueden referir los libros más citados, que de alguna manera se corresponden con los autores más citados. El ranking lo encabeza Skinner con dos de sus obras, y le sigue Ardila también con dos obras; la primera de autoría única y la segunda de autoría colectiva.

Libros más citados en el período 1969-1998

\begin{tabular}{|c|c|c|c|}
\hline & Autores & № Citas & $\%$ \\
\hline 1 & $\begin{array}{l}\text { Science and Human Behavior } \\
\text { (Skinner, B. F. 1953) }\end{array}$ & 26 & 0.43 \\
\hline 2 & $\begin{array}{l}\text { The Behavior of Organisms } \\
\text { (Skinner, B. F. 1938) }\end{array}$ & 19 & 0.32 \\
\hline 3 & $\begin{array}{l}\text { Psicología del Aprendizaje (Ar- } \\
\text { dila, R. 1970) }\end{array}$ & 18 & 0.30 \\
\hline 4 & $\begin{array}{l}\text { Análisis Experimental del } \\
\text { Comportamiento: la contribu- } \\
\text { ción latinoamericana (Ardila, } \\
\text { R. 1974) }\end{array}$ & 16 & 0.27 \\
\hline 5 & $\begin{array}{l}\text { Annals of New York Academy } \\
\text { of Sciences }\end{array}$ & 14 & 0.23 \\
\hline 6 & $\begin{array}{l}\text { Síntesis Experimental del Com- } \\
\text { portamiento (Ardila, R. 1988) }\end{array}$ & 12 & 0.20 \\
\hline 7 & $\begin{array}{l}\text { Tactics of Scientific Research } \\
\text { (Sidman, M. 1960) }\end{array}$ & 11 & 0.18 \\
\hline 8 & $\begin{array}{l}\text { Handbook of the Psychology of } \\
\text { Aging (Birren y Schaie.1977) }\end{array}$ & 11 & 0.18 \\
\hline 9 & $\begin{array}{l}\text { Annals of Dyslexia } \\
\text { Schedules of Reinforcement }\end{array}$ & 10 & 0.16 \\
\hline 10 & $\begin{array}{l}\text { (Ferster, C. B. y Skinner, B. F. } \\
\text { 1957) }\end{array}$ & 10 & 0.16 \\
\hline 11 & $\begin{array}{l}\text { Técnicas de Modificación de } \\
\text { Conducta (Ribes, E. 1972) }\end{array}$ & 10 & 0.16 \\
\hline 12 & $\begin{array}{l}\text { Annals of Psychology } \\
\text { Diagnostic and statistical }\end{array}$ & 9 & 15 \\
\hline 13 & $\begin{array}{l}\text { Manual of Mental Disorders } \\
\text { (DSM) }\end{array}$ & 8 & 0.13 \\
\hline 14 & $\begin{array}{l}\text { Principles of Behavior Modifi- } \\
\text { cation (Bandura, A. 1969) }\end{array}$ & 8 & 0.13 \\
\hline 15 & $\begin{array}{l}\text { The Structure of Scientific Re- } \\
\text { volutions (Kuhn, T. S. 1962) }\end{array}$ & 8 & 0.13 \\
\hline
\end{tabular}

Fuente: López López y Calvache (1998).

Es interesante destacar, que dentro del conjunto de las revistas más citadas durante las tres primeras décadas, la RLP no sólo es una de las publicaciones más refrenciadas, sino también la única editada en castellano (López López \& Calvache, 
1998). El ranking de las publicaciones más citadas en los artículos supone el siguiente orden:

Revistas más citadas en el período 1969-1998

\begin{tabular}{llcc}
\hline \multicolumn{1}{c}{ Revistas } & № & \% \\
\hline 1 & Revista Latinoamericana de Psico- & 244 & 2.81 \\
& logía & 211 & 2.43 \\
2 & American Psychologist & 157 & 1.81 \\
3 & Science & 153 & 1.76 \\
4 & Psychological Bulletin & 143 & 1.64 \\
5 & Journal Personality and Social Psy- & \\
& chology & 123 & 1.41 \\
6 & Psychological Review & 111 & 1.28 \\
7 & Journal of Comparative and Physio- & \\
& logical Psychology & 111 & 1.28 \\
8 & Journal of Consulting and Clinical & \\
& Psychology & 99 & 1.14 \\
9 & Behavior Therapy & 94 & 1.08 \\
10 & Journal of Abnormal and Social & 83 & 0.95 \\
& Psychology & 81 & 0.93 \\
11 & Psychosomatic Medicine & 78 & 0.89 \\
12 & Psychophysiology & 73 & 0.84 \\
13 & Behaviour Research and Therapy \\
14 & Archives of General Psychiatry \\
15 & Behavioral Pharmacology & 55 & 0.63 \\
\hline
\end{tabular}

Fuente: López López y Calvache (1998).

La RLP se encuentra indexada en diversas bases de datos regionales e internacionales. Durante sus años de existencia, fue una de las pocas revistas de psicología editada en castellano que ha logrado pertenecer a las bases de datos más importantes del mundo científico. Estas bases y otros sistemas de catalogación son fuentes de información científica de primera línea, que incluyen diversas revistas científicas, si han sido evaluadas satisfactoriamente de acuerdo a ciertos estándares de calidad. Como la RLP siempre ha mantenido buenos estándares de calidad, no fue difícil que se haya incluido y mantenido en las siguientes bases de datos.
Indexaciones de la RLP

ISI - Thomson Reuters
PsycInfo
Scopus
PsicoDoc
Publindex
Scielo
Redalyc
Pepsic
Psychological Abstracts
Biological Abstracts
Informe Académico
Dialnet
Bireme
Index Medicus Latinoamericano
Dietrich's Index Philosophicus
IBZ - Internationale Bibliographie der Geistes - und
- Sozialwissenschaftlichen
Zeitschriftenliteratur
Internationale bibliographie der Rezensionen
Wissenschaftlicherliteratur
Family and Society Studies Database
Ulrich's Internacional Periodicals Directory

Fuente: RLP (2010).

Además de estar incluida en estas importantes bases de datos científicas, la RLP se encuentra disponible en formato digital, con acceso abierto a todo el contenido desde el primer número. En algunos de los portales de revistas, como REDALYC ${ }^{11}$, SciELO ${ }^{12}$ o DIALNET ${ }^{13}$, se puede tener acceso directo a su información. Estos portales, además de evaluar la inclusión de las revistas científicas con base en ciertos estándares de calidad, cumplen con un importante rol en la difusión y visibilidad del conocimiento científico en el suelo iberoamericano.

Ciertamente, la disponibilidad full text de todo los artículos de las revistas, representa un importante avance en el contexto de apertura y democratización del conocimiento; al mismo tiempo, contribuye con la preservación de la memoria histórica de la ciencia. Es evidente que la función principal de las revistas científicas, es contribuir a

11 http://redalyc.uaemex.mx/

12 www.scielo.org/php/index.php

13 http://dialnet.unirioja.es/ 
la difusión de nuevos conocimientos; sin embargo, no debe descuidarse el importante papel que cumplen en el cuidado del patrimonio histórico y el acervo del conocimiento.

Con todo, las instituciones científicas y de educación superior también cumplen con un importante rol en la producción y difusión del conocimiento. Si bien los autores de los trabajos científicos son responsables por el nivel de su producción, no obstante, esa producción no sería posible sin el ámbito de trabajo que ofrecen las instituciones para desarrollar las investigaciones. Por tanto, desde que la actividad científica se ha institucionalizado, no es posible pensar aisladamente al investigador de su contexto institucional de producción. En este sentido, podemos indicar las entidades que más representadas han estado, durante los 30 primeros años de la RLP, a partir de la filiación institucional de sus autores.

Instituciones más representadas en el período 1969. 1998

\begin{tabular}{lcc}
\hline \multicolumn{1}{c}{ Institución } & № Arts. & $\%$ \\
\hline $\begin{array}{l}\text { Universidad Nacional Autónoma } \\
\text { de México (UNAM) }\end{array}$ & 47 & 7.0 \\
$\begin{array}{l}\text { Universidad de los Andes (Colom- } \\
\text { bia) }\end{array}$ & 30 & 4.47 \\
Universidad Nacional de Colombia & 25 & 3.72 \\
$\begin{array}{l}\text { Universidad de Buenos Aires } \\
\text { Centro Caribeño de Estudios Pos- }\end{array}$ & 20 & 2.98 \\
graduados (Puerto Rico) & 17 & 2.53 \\
$\begin{array}{l}\text { Universidad Católica de Chile } \\
\text { Universidad Nacional de San Luis }\end{array}$ & 16 & 2.38 \\
$\begin{array}{l}\text { (Argentina) } \\
\text { Universidad de Barcelona (España) }\end{array}$ & 14 & 2.08 \\
$\begin{array}{l}\text { Universidad Autónoma de Barcelo- } \\
\text { na (España) }\end{array}$ & 12 & 1.93 \\
$\begin{array}{l}\text { Pontificia Universidad Javeriana } \\
\text { (Colombia) }\end{array}$ & 12 & 1.78 \\
$\begin{array}{l}\text { Universidad de Puerto Rico } \\
\text { University of California (USA) }\end{array}$ & 11 & 1.63 \\
$\begin{array}{l}\text { Centro Interdisciplinario de Inves- } \\
\text { tigación en Psicología Matemática y }\end{array}$ & & 1.49 \\
$\begin{array}{l}\text { Experimental (CIIPME-Argentina) } \\
\text { Otras instituciones }\end{array}$ & 10 & 1.49 \\
\hline
\end{tabular}

Fuente: López López y Calvache (1998).
La presencia de estas instituciones revela el carácter no sólo latinoamericano, sino también interamericano e iberoamericano que ha contenido la RLP, a lo largo de su historia (López López \& Calvache, 1998). Más allá de que la procedencia institucional de los autores destaca a las instituciones de México, Colombia, Argentina, Puerto Rico, Chile, USA y España, no es menos importante señalar que las instituciones del resto de los países también han estado representadas a través de las producciones de sus autores (65\%).

Visiblemente, se puede reconocer en la RLP una revista con proyección internacional. No obstante, existen diversos criterios de internacionalidad de una publicación científica, tales como: idiomas de publicación, acceso por Internet, cumplimiento de normas de escritura o estilo, factor de impacto, inclusión en bases de datos, composición del comité editorial, procedencia de los autores, el título de la revista, entre otros (Zych \& BuelaCasal, 2007). Incluso, con base en estos criterios, se ha construido un índice de internacionalidad, para valorar cuán internacionales son las publicaciones científicas en psicología (Buela-Casal et al., 2007).

En el caso de la RLP, los análisis más recientes no sólo ratifican buenos criterios de internacionalidad, sino, además, buenos niveles de internacionalidad con respecto a otras publicaciones de psicología en el plano iberoamericano (véanse Villalobos \& Puertas, 2007; Zych \& Buela-Casal, 2007, 2009, 2010).

Entretanto, la colaboración científica ha tenido un franco crecimiento en los últimos años, y este crecimiento se ha cotejado en la RLP. En las dos primeras décadas, hubo una tendencia mayor a publicar trabajos de una sola autoría, mientras que, en la tercera década, aumentaron los trabajos firmados por más de dos autores (López López \& Calvache, 1998). Esto último, se confirma en la cuarta década, donde se ha registrado un mayor protagonismo de la autoría conjunta (Gutiérrez et al., 2009). En cierto sentido, este indicador esgrime la tendencia hacia una mayor colaboración en la investigación científica y en la producción de conocimientos, lo que contrasta con otros períodos históricos de la ciencia. 
La nacionalidad de los autores que más han publicado, generalmente se corresponde con aquellos países donde existe una extensa tradición en investigación científica en psicología (López López \& Calvache, 1998). Obviamente, el autor con más presencia de contribuciones es Rubén Ardila, con un total de 16 trabajos publicados. Sin embargo, no es menos frecuente la presencia reiterada de otros autores provenientes de diversos países.

Autores de mayor productividad en el período 1969. 2008

\begin{tabular}{llc}
\hline \multicolumn{1}{c}{ Nombre y Apellido } & \multicolumn{1}{c}{ País } & № arts. \\
\hline Rubén Ardila & Colombia & 16 \\
Luis Bravo Valdivieso & Chile & 11 \\
Mauricio Papini & Estados Unidos & 9 \\
Rogelio Díaz Guerrero & México & 9 \\
Alba Mustaca & Argentina & 8 \\
Stefano Vinaccia & Colombia & 7 \\
Ramón Bayés & España & 7 \\
Alejandro Cantón Dutari Panamá & 7 \\
Rosalía Montealegre & Colombia & 7 \\
Federico León & Perú & 6 \\
Alfonso Martínez Taboas & Puerto Rico & 6 \\
Ángel Rodríguez Kauth & Argentina & 6 \\
Ramón León & Perú & 6 \\
Germán Gutiérrez & Colombia & 5 \\
Pedro Solís Cámara & México & 5 \\
Josef Brožek & Estados Unidos & 5 \\
Fabio Sabogal & Estados Unidos & 5 \\
\hline
\end{tabular}

Fuente: Gutiérrez, Pérez-Acosta y Plata-Caviedes (2009).

El único país que tiene una gran tradición investigativa y una gran cantidad de psicólogos, pero que no aparece representado, es Brasil. Por supuesto que esta ausencia no significa que los autores brasileños no hayan elegido a la RLP, para volcar sus producciones. De hecho, no sólo se publicaron varias contribuciones de este país (Vera et al., 1991), sino, además, el comité editorial siempre estuvo integrado por algún representante de Brasil.

Es importante consignar que la ausencia o más bien la baja participación de la producción brasileña, no sólo se registra en la RLP, sino, también, en el contexto más general de la ciencia en la región. $\mathrm{Al}$ respecto, cabe señalar la creciente preocupa- ción que existe entre los cientistas, por mejorar la visibilidad e impacto de la producción originada en su geografía.

Brasil es un país que cuenta con una voluminosa producción científica; sin embargo, esa productividad tiene muy poco impacto en el escenario científico internacional (Bizarro, 2007). Sucede que los cientistas brasileños privilegian las referencias de autores foráneos en detrimento de su propia producción (Sampaio, 2008). Aun cuando sea altamente discutible y se haya tratado de mejorar, la definición conceptual y operativa de lo que se considera el factor de impacto (BuelaCasal, 2003; García, del Río \& Ramírez, 2002; Garfield, 2003; Navarrete-Cortés, Fernández-López, López-Baena, Quevedo-Blasco \& Buela-Casal, 2010; Pinto \& Andrade, 1999), lo cierto es que la producción brasileña tiende a quedar rezagada en los índices de citación.

Además, como es sabido, los autores brasileños son más propensos a publicar sus trabajos en inglés que en español, como segunda opción al portugués. Tal vez, por esta razón, los investigadores brasileños hayan pensado en otras revistas diferentes a la RLP, para publicar sus trabajos. En este punto, el predominio de la lengua inglesa en la ciencia contemporánea, parece conspirar a favor del escaso reconocimiento de los medios de divulgación científicas en la región.

Por otra parte, los investigadores brasileños han participado muy escasamente en el circuito de la producción científica latinoamericana (Koller, 2009). Recién en los últimos años, mayoritariamente en el ámbito del Mercosur y a propósito de una regionalización e internacionalización de la ciencia y la educación superior, se ha notado un incremento de la participación brasileña en la psicología de la región.

A raíz de toda esta situación, se han llevado a cabo diversas políticas y estrategias, para dar mayor protagonismo a la ciencia brasileña que, en el caso de la psicología, se ha traducido en los siguientes emprendimientos: $\mathrm{BSV}-\mathrm{Psi}^{14}$, PeP-

14 Biblioteca Virtual em Saúde - Psicologia. www.bvs-psi.org.bt 
$\mathrm{SIC}^{15}$ y ABECiP ${ }^{16}$. No obstante, también se han realizado connotados esfuerzos a nivel regional desde la creación del LATINDEX ${ }^{17}$, SciELO ${ }^{18}$, REDALYC $^{19}$ y diversos encuentros de editores y reuniones de cooperación científica (Buela-Casal \& López López, 2005; Calleja \& Albornoz, 2006; Cetto \& Alonso, 1999; Cetto \& Hillerud, 1995; Haupt, 2000; Hoyos, 1995).

Todo parece apuntar hacia una mayor consciencia, por parte de las instituciones científicas, los organismos gubernamentales, las agencias de financiación y los propios investigadores, de la importancia de la visibilidad del conocimiento y el reconocimiento de la producción originada en la región.

Sería deseable que esto se siga ratificando en el futuro. De alguna forma, las iniciativas que se vienen sosteniendo son claves importantes, para augurar un mayor acercamiento entre los académicos de la región y un positivo reconocimiento de la propia producción. En esa dirección, la RLP puede seguir sirviendo de puente entre la producción y socialización del conocimiento psicológico generado en nuestras tierras.

\section{Consideraciones finales}

Durante el tiempo transcurrido de su existencia, la RLP se ha convertido en parte esencial de la historia de la psicología en América Latina. Metafóricamente, se podría decir que la RLP fue el Windows de la psicología latinoamericana. En sus páginas, no sólo es posible rastrear una importante cantidad de contribuciones psicológicas, sino, además, se pueden conmemorar múltiples acontecimientos de la psicología como ciencia y profesión.

15 Portal de Periódicos em Psicologia. http://scielo.bvs-psi.org.br/ scielo.php

16 Associação Brasileira de Editores Científicos em Psicologia, originada en 2004 y formalizada en 2006 (Bizarro, 2006).

17 El Sistema Regional de Información en Linea para Revistas Científicas de América Latina, el Caribe, España y Portugal fue gestado en 1997 en México (véanse Cetto, 1998, 1999; Cetto, Alonso \& Rovalo, 1999).

18 Scientific Electronic Library Online. www.scielo.org/php/index.php

19 Red de Revistas Científicas de América Latina y el Caribe, España y Portugal. http://redalyc.uaemex.mx/
Muy pocas revistas pueden igualar la trayectoria y calidad de la RLP, máxime cuando se sabe que, en la historia de las revistas latinoamericanas de psicología, se han presenciado varias muertes prematuras e infelices discontinuidades.

Reconociendo el destacado papel de la RLP en la psicología hispanoamericana, solamente resta augurarle un próspero futuro. Ojalá que siga cumpliendo con ese importante rol de visualizar la producción psicológica latinoamericana.

\section{Referencias}

Aguilera, G. (2006). Proceso de estructuración e institucionalización del campo académico del desarrollo humano en el ITESO (1975-2005). Tesis doctoral no publicada, Instituto Tecnológico de Estudios Superiores de Occidente, México, DF, México.

Amil, A. \& Jardón, M. (2007). Dos publicaciones argentinas y su relación con dos hitos fundamentales en la historia de la psicología (1957-1962). Memorias de las XIV Jornadas de Investigación y Tercer Encuentro de Investigadores en Psicología del Mercosur, 3, 312-313.

Anónimo (1998). Biografías publicadas. Revista Latinoamericana de Psicología, 30 (3), 433-434.

Ardila, R. (1969a). Desarrollo de la psicología latinoamericana. Revista Latinoamericana de Psicología, 1 (1), 63-71.

Ardila, R. (1969b). Presentación. Revista Latinoamericana de Psicología, 1 (1), v-vi.

Ardila, R. (1973). Editorial. Revista Latinoamericana de Psicología, 5 (3), 253.

Ardila, R. (1978). Los diez primeros años de la Revista Latinoamericana de Psicología. Revista Latinoamericana de Psicología, 10 (3), 321-326.

Ardila, R. (1979). Editorial. Revista Latinoamericana de Psicología, 11 (1), 5-6.

Ardila, R. (1986a). La psicología en América Latina: pasado, presente y futuro. México: Siglo XXI.

Ardila, R. (1986b). La Revista Latinoamericana de Psicología y su papel en el desarrollo de la psicología hispanoparlante. Revista Latinoamericana de Psicología, 18 (3), 485-492. 
Ardila, R. (1988). Los 20 primeros años de la Revista Latinoamericana de Psicología. Revista Latinoamericana de Psicología, 20 (3), 401-406.

Ardila, R. (1994). Autobiografía de un psicólogo latinoamericano. Revista de Historia de la Psicología, 15 (1-2), 17-50.

Ardila, R. (1998). La Revista Latinoamericana de Psicología: los primeros 30 años. Revista Latinoamericana de Psicología, 30 (3), 393-400.

Ardila, R. (1999). Scientific publishing in Latin America: The case of journals in the behavioral and social sciences. En A. Cetto \& O. Alonso (Comps.), Revistas científicas en América Latina (pp. 207-225). México: ICSU/UNAM/CNCT/FCE.

Ardila, R. (2004). Editorial. Revista Latinoamericana de Psicología, 36 (1), v.

Ardila, R. (2007). Editorial. Revista Latinoamericana de Psicología, 39 (1), 7-8.

Bizarro, L (2006). Criação da ABECiP. Psicologia: Reflexão e Crítica, 19 (2), iii.

Bizarro, L (2007). Citar para ser citado. Psicologia: Reflexão e Crítica, 20 (2), iii.

Buela-Casal, G. (2003). Evaluación de la calidad de los artículos y de las revistas científicas: propuesta del factor impacto ponderado y de un índice de calidad. Psicothema, 15 (1), 23-35.

Buela-Casal, G. \& López López, W. (2005). Evaluación de las revistas científicas iberoamericanas de psicología. Iniciativas y estado actual. Revista Latinoamericana de Psicología, 37 (1), 211-217.

Buela-Casal, G., Zych, I., Sierra, J. \& Bermúdez, M. (2007). The internationality index of the spanish psychology journals. Revista Internacional de Psicología Clínica y de la Salud, 7 (3), 899-910.

Calvache, O., López López, W. \& Mayorga, E. (2002). Psicología clínica: 20 años de la revista Avances en Psicología Clínica Latinoamericana. Avances en Psicología Clínica Latinoamericana, 20, 11-30.

Calleja, B. \& Albornoz, M. (Comps.). (2006). Diálogo entre editores científicos iberoamericanos. Buenos Aires: CAICYT/CONICET.

Carpintero, H. \& Peiró, J. (Comps.). (1981). Psicología contemporánea. Teoría y métodos cuantitativos para el estudio de su literatura científica. Valencia: Alfaplus.

Centofanti, R. (1982). Radecki e a psicologia no Brasil. Psicologia: Ciência e Profissão, 3 (1), 3-50.
Cetto, A. (1998). Ciencia y producción científica en América Latina. El proyecto Latindex. International Microbiology, 1 (3), 181-182.

Cetto, A. (1999). Introducción. En A. Cetto \& O. Alonso (Comps.), Revistas científicas en América Latina (pp. 17-28). México: ICSU/UNAM/ $\mathrm{CNCT} / \mathrm{FCE}$.

Cetto, A., Alonso, O. \& Rovalo, L. (1999). Latindex, a dos años de su concepción. En A. Cetto \& O. Alonso (Comps.), Revistas científicas en América Latina (pp. 245-257). México: ICSU/UNAM/ CNCT/FCE.

Cetto, A. \& Hillerud, K. (Comps.). (1995). Publicaciones cientificas en América Latina. México: ICSU/ UNESCO/AIC/UNAM/FCE.

Cetto, A. \& Alonso, O. (Comps.). (1999). Revistas científicas en América Latina. México: ICSU/UNAM/ CNCT/FCE.

Dueñas, J. (2005). Breve reseña histórica de la ciencia psicológica cubana. Revista Cubana de Psicología, 22 (1), 56-61.

Filippo, D. \& Fernández, M. (2002). Bibliometría: importancia de los indicadores bibliométricos. El estado de la ciencia: principales indicadores de ciencia y tecnología iberoamericanos / interamericanos. Buenos Aires: RICYT.

Flórez, L. (Comp.). (2003). El legado de Rubén Ardila. Psicología: de la biología a la cultura. Bogotá: Universidad Nacional de Colombia.

Gallegos, M. (2009). Movimiento y participación estudiantil en la psicología latinoamericana: consideraciones históricas y futuras perspectivas. Avances en Psicología Latinoamericana, 27 (1), 33-60.

García, J. (2007). La psicología en Paraguay y el problema de la determinación de los pioneros. Revista Intercontinental de Psicología y Educación, 9 (2), 113-146.

García, J. (2008). Ramón Indalecio Cardozo como pionero de la psicología en el Paraguay. Revista Interamericana de Psicología, 42 (1), 171-180.

García, E., del Río, J. \& Ramírez, A. (2002). Análisis de la relevancia de las revistas latinoamericanas a través de un factor de impacto renormalizado. Revista Española de Documentación Científica, 25 (4), 467-476. 
Garfield, E. (2003). The meaning of the impact factor. Revista Internacional de Psicología Clínica y de la Salud, 3 (2), 363-369.

González, P. (1999). Revista Colombiana de Psicología (1992-1999). Revista Colombiana de Psicología, 9 , 139-141.

Guedes, M. \& Campos, R. (1999) (Comps.), Estudos em história da Psicologia. São Paulo: Educ.

Gutiérrez, G. (2008). Editorial. Revista Latinoamericana de Psicología, 40 (2), 209-210.

Gutiérrez, G., Pérez-Acosta, A. \& Plata-Caviedes, T. (2009). Desarrollo histórico de una publicación científica: cuarenta años de la Revista Latinoamericana de Psicología. Revista Latinoamericana de Psicología, 41 (3), 413-428.

Haupt, C. (2000). Las revistas científicas latinoamericanas: su difusión y acceso a través de bases de datos. Biblioteca Universitaria, 3 (2), 122-127.

Hoyos, N. (1995). Estado actual y perspectivas de las revistas científicas en la región Andina. En A. Cetto $\&$ K. Hillerud (Comps.), Publicaciones científicas en América Latina. México: ICSU/UNESCO/AIC/ UNAM/FCE.

Kirsch, U. \& Rodríguez Sturla, P. (2006). Presencia de la psicología en la revista Acta (1957-1962). Memorias de las XIII Jornadas de Investigación y Segundo Encuentro de Investigadores en Psicología del Mercosur, 3, 138-140.

Kirsch, U. \& Rojas Breu, G. (2007). Revista de Psiquiatría y Criminología - Revista Acta. Presencia del discurso psicológico. Continuidades y discontinuidades. Memorias de las XIV Jornadas de Investigación y Tercer Encuentro de Investigadores en Psicología del Mercosur, 3, 335-337.

Klappenbach, H., Barrozo, A., Cámara, S. \& López, N. (1999). Estudio bibliométrico de los primeros veinticinco años de Acta Psiquiátrica y Psicológica de América Latina (1954-1979). Acta Psiquiátrica y Psicológica de América Latina, 45 (4), 303-316.

Koller, S. (2009). Perspectivas e desafios para a disseminação em nível internacional para a produção brasileira. Revista Latinoamericana de Psicopatologia Fundamental, 12 (1), 13-16.

Lascurain, M., López, P. \& González, L. (1997). Psicología y bibliometría en España. Revista General de Información y Documentación, 7 (2), 95-107.
Lemos, F. (2005). Orientação profissional no Brasil: uma revisão histórica da produção científica. Revista Brasileira de Orientação Profissional, 6 (1), 15-24.

León, R. (1997). Rumbo al nuevo mundo: cuatro psicólogos de Europa Oriental en la historia de la psicología en América del Sur. Revista Latinoamericana de Psicología, 29 (1), 9-34.

López, L. (1992). Presentación. Revista Colombiana de Psicología, 1, 5.

López, P. (1996). Introducción a la bibliometría. Valencia: Promolibro.

López López, W. (2003). Rubén Ardila, una vida en la psicología internacional. En L. Flórez (Comp.), El legado de Rubén Ardila (pp. 188-193). Psicología: de la biología a la cultura. Bogotá: Universidad Nacional de Colombia.

López López, W. (2009). Editorial. De la cienciometría y los procesos de valoración de la producción intelectual. Universitas Psychologica, 8 (2), 291-292.

López López, W. \& Calvache, O. (1998). La psicología de habla hispana: 30 años de la Revista Latinoamericana de Psicología. Revista Latinoamericana de Psicología, 30 (3), 401-427.

Macías-Chapula, C. (1998). El papel de la infometría y de la cienciometría y su perspectiva nacional e internacional. Ciência da Informação, 27 (2), 35-41.

Navarrete-Cortés, J., Fernández-López, J., López-Baena, A., Quevedo-Blasco, R. \& Buela-Casal, G. (2010). Global psychology: A bibliometric analysis of Web of Science publications. Universitas Psychologica, 9 (2), 553-567.

Pérez-Acosta, A. (2007). Rubén Ardila recibe premio de la APA por contribuciones al Avance Internacional de la Psicología. Revista Latinoamericana Psicología, 39 (3), 669-671.

Pérez-Acosta, A. (2008). Los primeros cuatro años de la nueva época (2004-2007). Avances en Psicología Latinoamericana, 26 (1), 5-6.

Pinto, A. \& Andrade, J. (1999). Fator de impacto de revistas científicas: qual o significado deste parâmetro?. Química Nova, 22 (3), 448-453.

Quiñones, E., Vera, J., Sánchez, A. \& Pedraja, M. (1991). La segunda década de la Revista Latinoamericana de Psicología (1979-1988): una aproximación objetiva a la psicología en Latinoaméri- 
ca. Revista Latinoamericana de Psicología, 23 (2), 155-181.

Revista Latinoamericana de Psicología. (2010). Indexaciones. Disponible en www.revistalatinoamericanadepsicologia.com

Rossi, L. (2006). El diseño de las publicaciones periódicas en Argentina. Memorias de las XIII Jornadas de Investigación y Segundo Encuentro de Investigadores en Psicología del Mercosur,3, 156-158.

Rossi, L. (2007). Publicaciones periódicas en Argentina: producción discursiva e institucionalización. Referencias a la psicología. Anuario de Investigaciones, 14, 157-164.

Rossi, L. (2008). Redes discursivas, políticas e institucionales en las publicaciones periódicas en Argentina (1902-1962). Su influencia en al conformación del discurso psicológico. Anuario de Investigaciones, 15, 161-168.

Rousseau, R. (1997). Indicadores biliométricos y econométricos en la evaluación de instituciones científicas. Ciência da Informação, 27 (2), 50-50.

Salas, G. \& Lizama, E. (2009). Historia de la psicología en Chile. 1889-1981. La Serena: Universidad de La Serena.

Sampaio, M. (2008). Citações a periódicos na produção científica de psicologia. Psicologia: Ciência e Profissão, 28 (3), 425-465.
Sanabria, J. (1998). Los números monográficos de la Revista Latinoamericana de Psicología en sus 30 años. Revista Latinoamericana de Psicología, 30 (3), 429-432.

Sandoval, M. (2009). Editorial. Revista Latinoamericana de Psicología, 41 (3), 395-397.

Vera, J., Quiñones, E., Sánchez, A. \& Pedraja, M. (1991). La visibilidad de autores hispanoparlantes y lusoparlantes a través de la Revista Latinoamericana de Psicología (1979-1988). Revista de Historia de la Psicología, 12 (3-4), 307-318.

Villalobos, F. \& Puertas, R. (2007). Impacto de internacionalidad de tres revistas iberoamericanas en revistas de psicología en España. Revista Latinoamericana de Psicología, 39 (3), 593-608.

Zych, I. \& Buela-Casal, G. (2007). Índice de internacionalidad de las revistas iberoamericanas incluidas en la Web of Science. Revista Mexicana de Psicología, 24 (1), 15-22.

Zych, I. \& Buela-Casal, G. (2009). The internationality index: Application to Revista Latinoamericana de Psicología. Revista Latinoamericana de Psicología, 41 (3), 401-412.

Zych, I. \& Buela-Casal, G. (2010). Internacionalidad de las revistas de psicología multidisciplinar en Iberoamérica e incluidas en Web of Science. Universitas Psychologica, 9 (1), 27-34. 